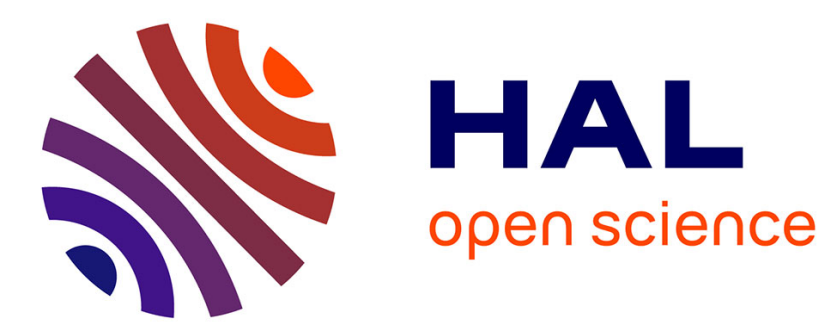

\title{
Introducing the pro-coagulant contact system in the numerical assessment of device-related thrombosis
}

Rodrigo Méndez Rojano, Simon Mendez, Franck Nicoud

\section{To cite this version:}

Rodrigo Méndez Rojano, Simon Mendez, Franck Nicoud. Introducing the pro-coagulant contact system in the numerical assessment of device-related thrombosis. Biomechanics and Modeling in Mechanobiology, 2018, 17 (3), pp.815-826. 10.1007/s10237-017-0994-3 . hal-01826601

\section{HAL Id: hal-01826601 https://hal.science/hal-01826601}

Submitted on 29 Jun 2018

HAL is a multi-disciplinary open access archive for the deposit and dissemination of scientific research documents, whether they are published or not. The documents may come from teaching and research institutions in France or abroad, or from public or private research centers.
L'archive ouverte pluridisciplinaire HAL, est destinée au dépôt et à la diffusion de documents scientifiques de niveau recherche, publiés ou non, émanant des établissements d'enseignement et de recherche français ou étrangers, des laboratoires publics ou privés. 


\title{
Introducing the pro-coagulant contact system in the numerical assessment of device-related thrombosis
}

\author{
Rodrigo Méndez Rojano • Simon Mendez • Franck Nicoud
}

Received: date / Accepted: date

\begin{abstract}
Thrombosis is a major concern in bloodcoated medical devices. Contact activation, which is the initial part of the coagulation cascade in device-related thrombosis, is not considered in current thrombus formation models. In the present study, pro-coagulant reactions including the contact activation system are coupled with a fluid solver in order to evaluate the potential of the contact system to initiate thrombin production. The biochemical/fluid model is applied to a backward facing step configuration, a flow configuration that frequently appears in medical devices. In contrast to the in vivo thrombosis models in which a specific thrombotic zone (injury region) is set a priori by the user to initiate the coagulation reaction, a reactive surface boundary condition is applied to the whole device wall. Simulation results show large thrombin concentration in regions related to recirculation zones without the need of an a priori knowledge of the thrombus location. The numerical results align well with the regions prone to thrombosis observed in experimental results reported in the literature. This approach could complement thrombus formation models that take into account platelet activity and thrombus growth to optimize a wide range of medical devices.
\end{abstract}

Keywords Thrombosis - Medical devices · Contact System · Hemodynamics

Rodrigo Méndez Rojano · Simon Mendez · Franck Nicoud Institut Montpelliérain Alexander Grothendieck,

CNRS, Univ. Montpellier

2 Place Eugène Bataillon, 34095 Montpellier Cedex 5, France

E-mail: rodrigo.mendez-rojano@umontpellier.fr

\section{Introduction}

The use of blood-coated devices is a common medical practice to treat several cardiovascular conditions. However, the poor thrombotic performance of some types of medical devices remains an issue in clinical practice (Chan et al. (2009); Mehra et al. (2014); Hastings et al. (2017)). For instance, thrombus formation may lead to device malfunction, thrombo-embolism or increased risk of a hemorrhagic stroke due to the anti-coagulant therapy. Thrombosis is a complex phenomenon that involves several processes such as coagulation reactions, platelet activation/aggregation, blood flow dynamics, among others. Furthermore, these mechanisms interact with each other at different time and spatial scales as explained in Furie and Furie (2008). When it comes to device-related thrombosis, the artificial wall of the device interacts with the thrombotic processes as pointed out by Gorbet and Sefton (2004). Wall protein adsorption (Vroman effect) drives contact activation that initiates the coagulation reactions. At the same time, platelets adhesion and activation take place (Jaffer et al. (2015)), constituting the platelet-fibrin clot that will form the stable thrombus.

In recent studies, computational fluid dynamics has been used to predict the flow in medical devices, whith the long-term objective of improving their thrombotic performance (Alemu et al. (2010); Piatti et al. (2015)). However, most of the thrombus formation models are dedicated to platelet activity or in-vivo situations in which no medical device is present. For instance, numerical studies that consider platelet activity are notably focused on the characterization of mechanical activation of platelets (Hellums (1994); Kroll et al. (1996); Jetsy et al. (2003); Nobili et al. (2008); Shadden and Hendabadi (2013)). In contrast, in-vivo models are based 
on a series of reaction equations modeling the coagulation cascade, from the exposure of tissue factor to the generation of thrombin (Sorensen et al. (1999); Kuharsky and Fogelson (2001); Fogelson and Guy (2008); Anand et al. (2008); Seo et al. (2016); Yazdani et al. (2017)). Generally, in vivo models, prescribe an injury zone determined by the user producing tissue factor and thus, initiating the coagulation reactions (Biasetti et al. (2012); Seo et al. (2016)). This type of approach cannot be used for device-related thrombosis due to the lack of a priori knowledge of the thrombotic zone. For this reason, the models dedicated to evaluate the device thrombotic performance have focused on platelet activity. For instance, Goodman et al. (2005) developed a model to predict device-induced thrombosis based on platelet activation (agonist induced platelet activation as in Sorensen et al. (1999) and mechanical platelet activation by shear stress). Furthermore, the model accounts for platelet adhesion to artificial surfaces and thrombus growth by substantially increasing the fluid viscosity. Taylor et al. (2016) developed a macroscopic model that includes platelet activation and thrombus growth in regions related to high and low wall shear stress (WSS), respectively. Their model was derived from the work of Fogelson (1992) with some modifications to account for device-related thrombosis.

While platelet activity has been extensively considered, other major players like the complement and contact activation systems must be included in the modeling, as pointed out by Gorbet and Sefton (2004). Moreover, considering contact activation could provide significant information to platelet models since one mechanism of their activation is due to thrombin as explained in Fogelson and Neeves (2015), the latter being partially produced by reactions initiated by the contact system (Larsson et al. (2014)). To the best of our knowledge, the only model considering the interaction of an idealized flow configuration and the contact activation system is the one of Gregory and Basmadjian (1994). In their work, a steady-state solution of a kinetic model is analyzed. The coagulation reactions in this model include contact activation of factor $X I I$ and the common pathway of the coagulation cascade (until factor $\left.X I_{a}\right)$. The model results showed significant sensitivity to the flow dynamics and surface activity. A more recent kinetic model that considers the contact system in a platelet plasma kinetic network was developed by Chatterjee et al. (2010). Their model predicts the coagulation time of quiescent human blood samples without the action of tissue factor (TF). The results show that coagulation is due to the combined action of factors $X I I_{a}$ and $X I_{a}$.

In parallel, recent clinical studies have pointed at the contact activation phase as a promising therapeutic target providing an anticoagulant strategy without increasing the risk of bleeding (Jaffer et al. (2015); Larsson et al. (2014)). A model that incorporates the contact activation system and the coagulation reactions until thrombin formation coupled with a realistic representation of flow dynamics is thus needed. To this respect, the objective of this study is to evaluate the potential of the contact system to initiate thrombin production. The role of the anionic phospholipids (platelet activity), fibrin formation and fluid-structure interaction of a growing thrombus were thus neglected, since these phenomena, although prominent in the general thrombus formation mechanism, are not specificaly involved in the surface production of thrombin. A surface-mediated thrombin production model is presented in a computational hemodynamics framework. The model accounts for interaction of the blood flow with the contact activation system and the rest of the blood coagulation cascade; it is detailed in Section 2, together with the numerical method. Furthermore, the model was applied to a device-characteristic flow configuration presented in the experimental work of Taylor et al. (2014). To this end, a specific boundary condition was developed to mimic the contact activation at the device surface. The results discussed in section 3 illustrate the potential of the approach.

\section{Materials and Methods}

\subsection{Governing equations}

\subsubsection{Fluid dynamics equations}

Flow dynamics is described by the incompressible Navier Stokes equations that read

$\nabla \cdot \mathbf{u}=0$

$\rho\left(\frac{\partial \mathbf{u}}{\partial t}+\mathbf{u} \cdot \nabla \mathbf{u}\right)=-\nabla p+\mu \nabla^{2} \mathbf{u}$

where $\mathbf{u}$ is the velocity field, $p$ is the pressure field, $\rho$ stands for the density and $\mu$ is the dynamic viscosity (both assumed constant-valued). The blood was considered as a Newtonian fluid (constant viscosity) since the objective of the present study was not focused on the effects due to Non-Newtonian behavior of blood.

\subsubsection{Convection-Diffusion-Reaction equations}

To account for the coagulation reactions, a set of evolution equations (one for each coagulation factor) is 
solved. The equation that captures the spatial and temporal evolution of the biochemical species is widely known as the convection-diffusion-reaction equation

$\frac{\partial C_{i}}{\partial t}+\nabla \cdot\left(C_{i} \mathbf{u}\right)=\nabla \cdot\left(D_{i} \nabla C_{i}\right)+R_{i}$

where $C_{i}\left(\mathrm{M}=\mathrm{mol} \mathrm{L}^{-1}=10^{3} \mathrm{~mol} \mathrm{~m}^{-3}\right)$ and $D_{i}\left(\mathrm{~m}^{2} \mathrm{~s}^{-1}\right)$ are the molar concentration and diffusion coefficients for each species $i, R_{i}\left(\mathrm{M} \mathrm{s}^{-1}\right)$ is the reaction source term (computed according to the reactions rates presented in Table 1).

\subsection{Coagulation model}

A wide variety of models has been proposed to predict thrombin formation inside the blood stream (Anand et al. (2003); Leiderman and Fogelson (2011); Biasetti et al. (2012)). In those models, coagulation reactions start at the extrinsic pathway by the exposure of TF. In the present work, the presence of an artificial surface in contact with blood is accounted for by including the contact activation system (Basmadjian et al. (1997)). For this purpose the kinetic model of Chatterjee et al. (2010) is adopted. This set of reactions predicts thrombin production in the absence of exogenous $\mathrm{TF}$ and starts the coagulation reactions by the contact activation of factor $X I I$. Figure 1 shows a schematic of the coagulation model used for the simulations including the contact activation system and the rest of the coagulation cascade reactions.

In Table 1, the 26 reactions used are listed with their respective reaction rates. The types of reaction kinetics are of first order, second order and Michaelis-Menten. Reaction rates for the different reactions are listed in three different columns according to their type. The source terms $R_{i}$ appearing in Eq. 3 are deduced from the coagulation reactions. It is important to mention that the 53 reaction rates values do not consider the platelet activity that is also reported by Chatterjee et al. (2010).

Diffusion coefficients were estimated with the correlation of Young et al. (1980). The molecular weight of each chemical species is needed to estimate the diffusion coefficient along with the temperature and the dynamic viscosity. For the present study a temperature of $T=25^{\circ} \mathrm{C}$ and a dynamic viscosity of $\mu=3.71 \times 10^{-3}$ $\mathrm{Pa} \mathrm{s}$ were used. The correlation used considers a partial specific volume of $0.73 \mathrm{~cm}^{3} \mathrm{~g}^{-1}$ which is assumed to be common for all the species. The expression proposed by Young et al writes:

$D=8.34 \times 10^{-8}\left(T / \mu M^{1 / 3}\right)$
The diffusion values estimated with Eq. 4 are extremely small (of the order of $10^{-11} \mathrm{~m}^{2} \mathrm{~s}^{-1}$ ). If these values were to be used, numerical instabilities and large diffusion characteristic times would render the convergence to a steady state solution extremely difficult. This problem has been emphasized in studies using numerical models for coagulation. This difficulty is either overcome by refining the mesh which is hardly feasible except for 1D simulations, or by increasing artificially the diffusion coefficients as in Biasetti et al. (2012). Here, we follow the latter approach and multiply all diffusion coefficients by a factor of 1000 .

The main objective of this work is to evaluate the potential of the contact activation system to initiate thrombin formation. Consequently, the model did not include inhibitors, TF and fibrin-related reactions that are included in Chatterjee et al. (2010). Finally the model assumes an infinite number of binding sites (infinite platelet supply), as in Biasetti et al. (2012). The reader is referred to Kuharsky and Fogelson (2001); Anand et al. (2003) for a discussion on the role of a finite number of binding sites. It is important to note that the scope of this article is not to model the complete and complex dynamics of thrombus formation. A full model must include the role of anionic phospholipids, platelet adhesion and aggregation as in Leiderman and Fogelson (2011). Other important players for a robust model should be included such as fibrin production (Neeves et al. (2010)), coagulation inhibitors (Zhu (2007); Rice et al. (2016)) or thrombus growth (solid-fluid interaction) as model by Ngoepe and Ventikos (2016). Such a comprehensive model may provide a good prediction tool to contribute to the optimization of medical devices. However, the potential of the contact activation system to initiate coagulation reactions is better assessed by focusing on this mechanism alone, without including all the other phenomena relevant to thrombus formation. This is the methodology followed in this study.

\subsection{A boundary condition for the contact activation}

To model the contact activation of factor $X I I$, a wall boundary condition is derived from the species mass balance at the wall where protein adsorption takes place followed by contact activation. The balance is obtained by integrating Eq. 3 over a rectangular control volume $\Omega$ (which includes a volume of fluid and a volume of wall, see Fig. 2) of surface $\Sigma$. Using the divergence theorem to reformulate the conservative terms and integrating over $\Omega$ leads to: 


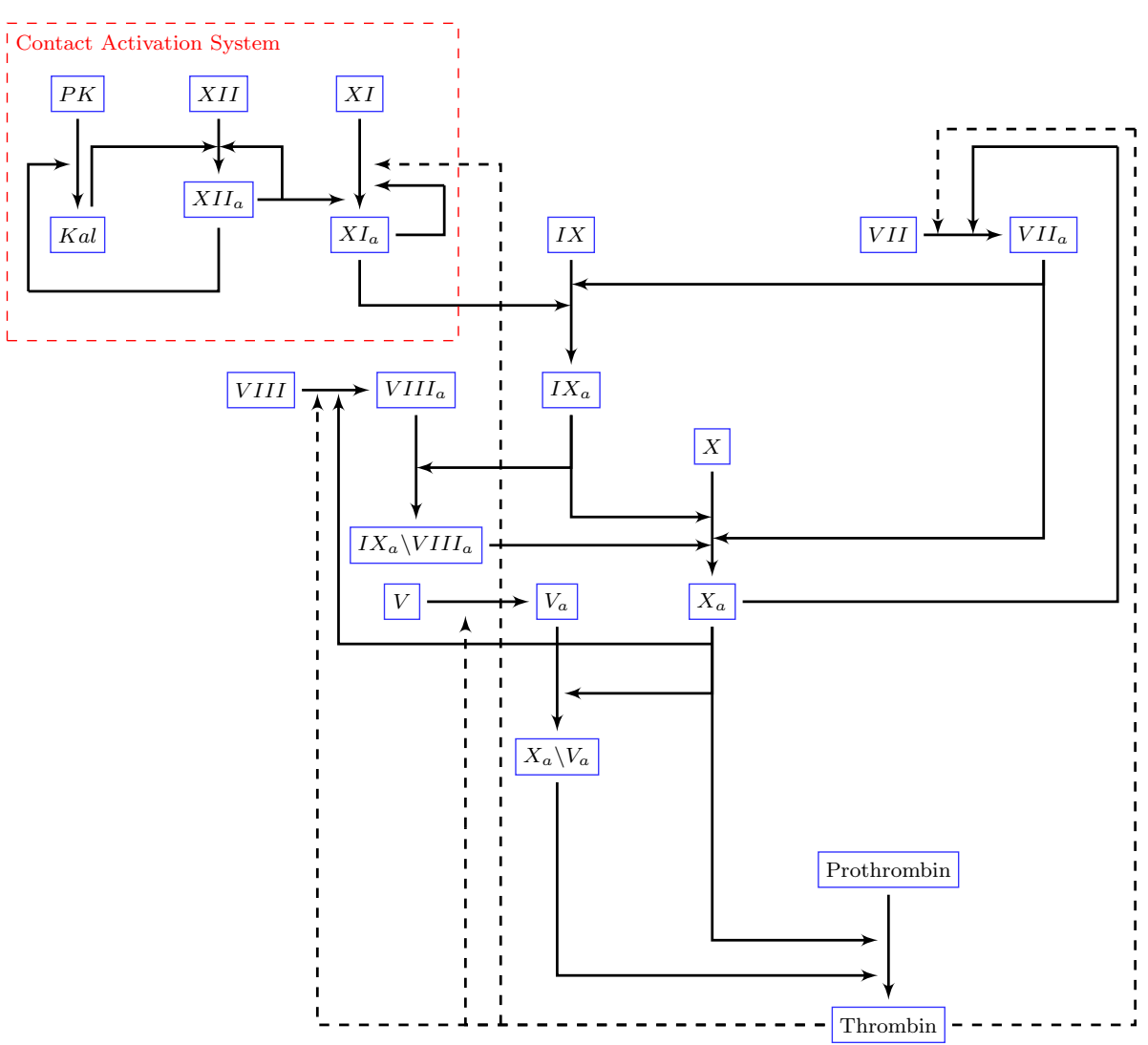

Fig. 1 Schematic of the coagulation reaction network. The kinetic network is inspired from the platelet-plasma model of Chatterjee et al. (2010) without considering TF, inhibitors and fibrinogen reactions. The dashed lines correspond to the feedback loop reactions related to thrombin.

\begin{tabular}{|c|c|c|c|c|}
\hline \# & Reaction & Forward $M^{-1} s^{-1}$ & Reverse $s^{-1}$ & Forward $s^{-1}$ \\
\hline 1 & $X a+V I I \rightarrow X a+V I I a$ & $k_{1}=1.3 \times 10^{7}$ & & \\
\hline 2 & $I I a+V I I \rightarrow I I a+V I I a$ & $k_{2}=2.3 \times 10^{4}$ & & \\
\hline 3 & $I I+X a \rightarrow I I a+X a$ & $k_{3}=7.5 \times 10^{3}$ & & \\
\hline 4 & $I I a+V I I I \rightarrow I I a+V I I I a$ & $k_{4}=2.0 \times 10^{7}$ & & \\
\hline 5 & $V I I I a+I X a \leftrightarrow I X a=V I I I a$ & $k_{5}=1.0 \times 10^{7}$ & $k_{6}=5.0 \times 10^{-3}$ & \\
\hline 6 & $I X a=V I I I a+X \leftrightarrow I X a=V I I I a=X \rightarrow I X a=V I I I a+X a$ & $k_{7}=1.0 \times 10^{8}$ & $k_{8}=1.0 \times 10^{-3}$ & $k_{9}=8.2$ \\
\hline 7 & $V I I I a \leftrightarrow V I I I a_{1} \cdot L+V I I I a_{2}$ & & $k_{10}=6.0 \times 10^{-3}$ & $k_{11}=2.2 \times 10^{4}$ \\
\hline 8 & $I X a=V I I I a=X \rightarrow V I I I a_{1} \cdot L+V I I I a_{2}+X+I X a$ & & $k_{12}=1.0 \times 10^{-3}$ & \\
\hline 9 & $I X a=V I I I a \rightarrow V I I I a_{1} \cdot L+V I I I a_{2}+I X a$ & & $k_{13}=1.0 \times 10^{-3}$ & \\
\hline 10 & $I I a+V \rightarrow I I a+V a$ & $k_{14}=2.0 \times 10^{7}$ & & \\
\hline 11 & $X a+V a \leftrightarrow X a=V a$ & $k_{15}=4.0 \times 10^{8}$ & $k_{16}=0.2$ & \\
\hline 12 & $X a=V a+I I \leftrightarrow X a=V a=I I \rightarrow X a=V a+m I I a$ & $k_{17}=1.0 \times 10^{8}$ & $k_{18}=103$ & $k_{19}=63.5$ \\
\hline 13 & $X a=V a+m I I a \rightarrow X a=V a+I I a$ & $k_{20}=1.5 \times 10^{7}$ & & \\
\hline $14^{*}$ & $X I I \rightarrow X I I a$ & & & $k_{s}=5.0 \times 10^{-7} \mathrm{~ms}^{-1}$ \\
\hline 15 & $X I I a+X I I \leftrightarrow X I I a=X I I \rightarrow X I I a+X I I a$ & $k_{22}=1 \times 10^{8}$ & $k_{23}=750$ & $k_{24}=3.3 \times 10^{-2}$ \\
\hline 16 & $X I I a+P K \leftrightarrow X I I a=P K \rightarrow X I I a+K$ & $k_{25}=1 \times 10^{8}$ & $k_{26}=3.6 \times 10^{3}$ & $k_{27}=40$ \\
\hline 17 & $X I I+K \leftrightarrow X I I=K \rightarrow X I I a+K$ & $k_{28}=1 \times 10^{8}$ & $k_{29}=45.3$ & $k_{30}=5.7$ \\
\hline 18 & $P K+K \rightarrow K+K$ & $k_{31}=2.7 \times 10^{4}$ & & \\
\hline 19 & $X I+I I a \leftrightarrow X I=I I a \rightarrow X I a+I I a$ & $k_{32}=1 \times 10^{8}$ & $k_{33}=5$ & $k_{34}=1.3 \times 10^{-4}$ \\
\hline 20 & $X I I a+X I \leftrightarrow X I I a=X I \rightarrow X I I a+X I a$ & $k_{35}=1.0 \times 10^{8}$ & $k_{36}=200$ & $k_{37}=5.7 \times 10^{-4}$ \\
\hline 21 & $X I a+X I \rightarrow X I a+X I a$ & $k_{38}=3.19 \times 10^{6}$ & & \\
\hline 22 & $X I a+I X \leftrightarrow X I a=I X \rightarrow X I a+I X a$ & $k_{39}=1.0 \times 10^{8}$ & $k_{40}=41.0$ & $k_{41}=7.7$ \\
\hline 23 & $I X a+X \leftrightarrow I X a=X \rightarrow I X a+X a$ & $k_{42}=1.0 \times 10^{8}$ & $k_{43}=0.64$ & $k_{44}=7.0 \times 10^{-4}$ \\
\hline 24 & $X a+V I I I \leftrightarrow X a=V I I I \rightarrow X a+V I I I a$ & $k_{45}=1.0 \times 10^{8}$ & $k_{46}=2.1$ & $k_{47}=0.023$ \\
\hline 25 & $V I I a+I X \leftrightarrow V I I a=I X \rightarrow V I I a+I X a$ & $k_{48}=1.0 \times 10^{8}$ & $k_{49}=0.9$ & $k_{50}=3.6 \times 10^{-5}$ \\
\hline 26 & $V I I a+X \leftrightarrow V I I a=X \rightarrow V I I a+X a$ & $k_{51}=1.0 \times 10^{8}$ & $k_{52}=210$ & $k_{53}=1.6 \times 10^{-6}$ \\
\hline
\end{tabular}

Table 1 Coagulation reactions. Kinetic parameters and coagulation reactions used in the simulations. Reaction 14 is taken into account only at the surface by means of the wall boundary condition. Parameter $k_{s}$ units $\left(\mathrm{m} \mathrm{s}^{-1}\right)$ correspond to a surface reaction; its value was obtained by adapting the volume reaction rate from Chatterjee et al. (2010).

$$
\begin{array}{r}
\int_{\Omega} \frac{\partial C_{i}}{\partial t} d \Omega+\int_{\Omega}-R_{i} d \Omega=\int_{\Sigma}\left(D_{i} \nabla C_{i}\right) \cdot \mathbf{n} d \Sigma \\
-\int_{\Sigma} C_{i} \mathbf{u} \cdot \mathbf{n} d \Sigma
\end{array}
$$

The reaction source term $R_{i}$ can be decomposed into the volume (blood-phase) reaction source term $\dot{\omega}_{i}$ and the surface source term $\dot{s}_{i}$ multiplied by a Dirac function $\delta$ (which is no zero at the device surface). The mass 


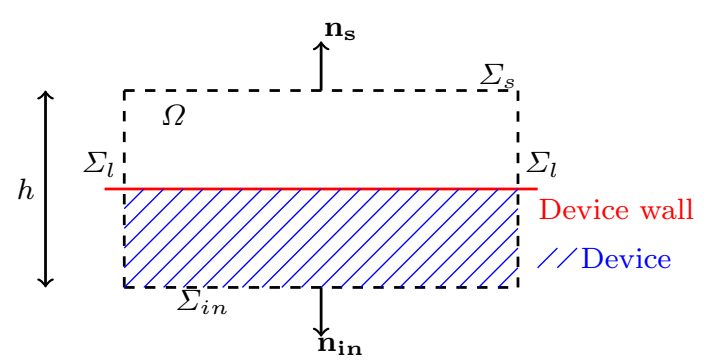

Fig. 2 Mass balance around the device wall. $\Omega$ Control of volume, $\Sigma$ Surface envelope of the control volume, Sub index in, $s$ and $l$ stand for the inferior, superior and lateral surfaces respectively, $h$ height of the control volume.

balance becomes:

$$
\begin{aligned}
\int_{\Omega} \frac{\partial C_{i}}{\partial t} d \Omega+\int_{\Omega}-\left(\dot{\omega}_{i}+\delta \dot{s}_{i}\right) d \Omega= & \int_{\Sigma}\left(D_{i} \nabla C_{i}\right) \cdot \mathbf{n} d \Sigma \\
& -\int_{\Sigma} C_{i} \mathbf{u} \cdot \mathbf{n} d \Sigma
\end{aligned}
$$

The surface $\Sigma$ can be separated in lateral, inferior and superior surfaces respectively $\Sigma=2 \Sigma_{l}+\Sigma_{i n}+\Sigma_{s}$. If the height $h$ tends to zero $(h \rightarrow 0)$ the volume integrals over $\Omega$ and the surface integrals over the lateral surfaces $\Sigma_{l}$ are equal to zero; the inferior surface $\Sigma_{i n}$ collapse with the device wall and the surface reaction source term can be transformed in a surface integral over the device wall thanks to the Dirac function. In addition the convection fluxes at the device surface are equal to zero (assuming the wall is at rest and impermeable), thus Eq.5 can be rewritten as:

$$
\begin{aligned}
-\int_{\Sigma_{s}}\left(D_{i} \nabla C_{i}\right) \cdot \mathbf{n}_{\mathbf{s}} d \Sigma_{s}= & \int_{\Sigma_{i n}}\left(D_{i} \nabla C_{i}\right) \cdot \mathbf{n}_{\mathbf{i n}} d \Sigma_{i n} \\
& +\int_{\Sigma_{i n}} \dot{s_{i}} d \Sigma_{i n}
\end{aligned}
$$

The diffusion flux inside the solid domain is zero since no species is present in the device itself; thus one can write:

$-\int_{\Sigma_{s}}\left(D_{i} \nabla C_{i}\right) \cdot \mathbf{n}_{\mathbf{s}} d \Sigma_{s}=\int_{\Sigma_{i n}} \dot{s}_{i} d \Sigma_{i n}$

A boundary condition can be obtained by applying Eq. 8 to factor $X I I_{a}$. The surface source term is obtained assuming an infinitely fast adsorption and an infinite number of binding sites for factor $X I I$, thus the reaction that takes places is the conversion of the near-wall factor $X I I$ (indexed by ${ }_{w}$ ) to $X I I_{a}$. Finally the following wall boundary condition is obtained:

$D_{X I I_{a}} \frac{\partial C_{X I I a}}{\partial n}=-k_{s} C_{X I I_{w}}$

where $k_{s}$ is a material property (surface reaction rate) and the direction $n$ is the normal direction pointing to the blood-phase of the domain. In this study the value published in Chatterjee et al. (2010) for the activation of factor XII in a perfectly mixed environment (volumetric reaction rate) was converted to a surface reaction rate $k_{s}$. From a straightforward dimensional analysis, one obtains $k_{s} \sim k_{v} \frac{V_{\text {batch }}}{S_{\text {batch }}}$ where $V_{\text {batch }}$ and $S_{\text {batch }}$ are the characteristic volume and the active surface of the experimental set up respectively. The surface reaction rate $k_{s}=5.0 \times 10^{-7} \mathrm{~ms}^{-1}$ is retrieved by using $V_{\text {batch }} \sim 10 \mu \mathrm{L}$ and $S_{\text {batch }} \sim 0.1 \mathrm{~cm}^{2}$ following the experimental values reported in Chatterjee et al. (2010).A range of values was also considered to investigate the effect of this physical quantity on the results (see section 3).

\subsection{Numerical implementation and verification}

Equations 1 and 2 were solved with a fourth-order finitevolume method on an unstructured grid, implemented in the in-house YALES2BIO ${ }^{1}$ solver. YALES2BIO is based on a massivel parallel finite volume solver for incompressible flows (Moureau et al. (2011)) and has been extensively validated for several biomedical applications at micro and macro scale (Chnafa et al. (2014b,a); Mendez et al. (2014); Sigüenza et al. (2017); Chnafa et al. (2016); Lanotte et al. (2016); Zmijanovic et al. (2017)). It uses a spatial fourth-order scheme with an explicit four-step Runge-Kutta scheme in time. The pressure term is handle with the Chorin's projectioncorrection method. Equation 3 was also solved using a fourth-order scheme in space and time for each species. Several test cases were developed to verify the implementation of Eq. 3 in YALES2BIO. Test cases with reactions of the Michaeles-Menten type (Michaelis et al. (2011)), as well as first and second order reactions were compared against analytic solutions. Furthermore, the results of the kinetic model introduced by Hockin et al. (2002) were reproduced. In this test case the reactions start with an initial condition of exogenous TF. This allows to validate the different types of reactions that are shared by the different coagulation kinetic schemes; for instance, reactions 1 to 13 in Table 1 are shared with the scheme of Hockin et al. (2002).

Moreover, a simple 1D diffusive test case involving a volume and a surface reaction (contact activation boundary condition) without convection was specifically designed during the course of this study. In this academical configuration illustrated in Fig. 3, a species $A$ can be either activated at the bottom wall and become $A_{a}$ (surface reaction rate $k_{s}$ ) or transformed into

\footnotetext{
1 http://www .math.univ-montp2.fr/ yales2bio/
} 
species $B$ in the flow domain (volume reaction rate $k_{v}$ ), whereas the upper wall acts like an infinite reservoir at concentration $C_{A}(h)=\alpha$. In the calculations the domain is $2 \mathrm{D}$, with periodic conditions in the directions tangential to the walls. The analytic steady state solution for the concentration of species $A$ reads:

$C_{A}(y)=\frac{\alpha \mathrm{e}^{\beta(h-y)}\left(k_{s}\left(\mathrm{e}^{2 \beta y}-1\right)+\beta D_{A}\left(1+\mathrm{e}^{2 \beta y}\right)\right)}{k_{s}\left(\mathrm{e}^{2 \beta h}-1\right)+\beta D_{A}\left(1+\mathrm{e}^{2 \beta h}\right)}$

where $k_{s}$ and $k_{v}$ are the surface and volume reaction rates, respectively, $\beta=\sqrt{\frac{k_{v}}{D_{A}}}$ and $h$ is the channel height. Table 2 shows the three different operating points that were used to verify the results.

\begin{tabular}{ccc}
\hline Cases & $k_{s} \mathrm{~m} \mathrm{~s}^{-1}$ & $k_{v} \mathrm{~s}^{-1}$ \\
\hline Case $_{1}$ & $1.0 \times 10^{-4}$ & $1.0 \times 10^{4}$ \\
Case $_{2}$ & $1.0 \times 10^{-4}$ & $1.0 \times 10^{3}$ \\
Case $_{3}$ & $1.0 \times 10^{-2}$ & $1.0 \times 10^{2}$ \\
\hline
\end{tabular}

Table 2 Operating points. The different set of parameters $\left(k_{s}\right.$ and $\left.k_{v}\right)$ used for the verification of the code. The corresponding steady state solutions are shown in Fig. 3.

Figure 3 shows the steady state solution of species $A$ for both the numerical and analytic cases. An excellent agreement is obtained for all of the operating points.
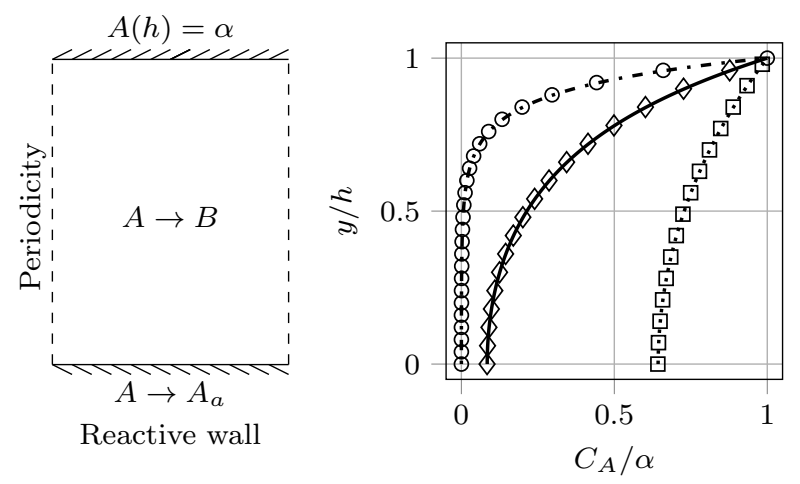

Fig. $31 \mathrm{D}$ reaction-diffusion test case. $C_{A}=\alpha=1.0 \mu \mathrm{M}$, initial volume concentration of all the species $C_{i}=0 \mathrm{M}$, diffusion coefficient of species $D_{A}=D_{B}=1.0 \times 10^{-2} \mathrm{~m}^{2} \mathrm{~s}^{-1}$, channel height $h=0.01 \mathrm{~m}$. Numerical results (Case ${ }_{1}$ o, Case $_{2} \diamond$, Case $\left._{3} \square\right)$ and analytic Eq. 10 data $\left(\right.$ Case $_{1} \cdot-\cdot-$, $\left.\mathrm{Case}_{2} \longrightarrow \mathrm{Case}_{3} \cdots \cdots\right)$.

\subsection{Backward facing step configuration}

Numerical simulations are performed in a backward facing step (BFS) configuration presented in Fig. 4, advancing the set of reactions presented in Table 1 . This type of configuration involves a flow separation that several medical devices could feature and which has been related to thrombus formation (Hastings et al. (2017); Taylor et al. (2014)). The step height $h_{s}=2.5$ $\mathrm{mm}$ and vessel height $H=10 \mathrm{~mm}$ match the experimental dimensions presented by Taylor et al. (2014).

The inlet and outlet conditions were placed at a distance of $10 \mathrm{H}$ to avoid boundary effects on the region of interest located downstream of the step: the expected recirculation zone for the flow regime considered (Reynolds number $R e=430$, based on the inlet channel height and the upstream bulk velocity) is $X r=8.9 h_{s}$ according the results of Kim and Moin (1985). The kinematic viscosity was set to a constant value $\nu=3.5 \times$ $10^{-6} \mathrm{~m}^{2} \mathrm{~s}^{-1}$.

Boundary and initial conditions. The inlet velocity was set to $U_{x}=0.2 \mathrm{~m} \mathrm{~s}^{-1}$, consistently with the experimental conditions described in Taylor et al. (2014). Table 3 shows the initial and inlet boundary conditions factors that were taken from Chatterjee et al. (2010) and which correspond to physiological values. The boundary and initial conditions for the rest of the factors were set to zero. One of the most important features of the simulation is the reactive surface boundary condition, Eq. 9. The boundary condition for the contact activation of factor $X I I$ was applied at the entire wall of the computational domain (see Fig. 4).

\begin{tabular}{cc}
\hline Factor & Concentration $(\mathrm{M})$ \\
\hline$V I I$ & $1.0 \times 10^{-8}$ \\
$V I I a$ & $1.0 \times 10^{-10}$ \\
$X$ & $1.6 \times 10^{-7}$ \\
$I X$ & $9.0 \times 10^{-8}$ \\
$I I$ & $1.4 \times 10^{-6}$ \\
$V I I I$ & $7.0 \times 10^{-10}$ \\
$V$ & $2.0 \times 10^{-8}$ \\
$X I I$ & $3.4 \times 10^{-7}$ \\
$P K$ & $4.5 \times 10^{-7}$ \\
$X I$ & $3.1 \times 10^{-8}$ \\
\hline
\end{tabular}

Table 3 Initial and inlet factor concentrations. Physiological values used in the simulations taken from Chatterjee et al. (2010).

Spatial and temporal resolution. Several grids were used to ensure that the simulations yield results independent from the grid used in the calculation. Table 4 shows the representative cell size and the number of elements used for the computations. The simulation time step is given by the Courant-Friedrichs-Levy stability condition which is define using the time step $\Delta t$, the local velocity field $u$ and the mesh size $\Delta x$ as $C F L=\frac{u \Delta t}{\Delta x} \leq 1$. The value used in the current simulations is $C F L=0.9$ which corresponds to a time step of the order $10^{-4} \mathrm{~s}$. 


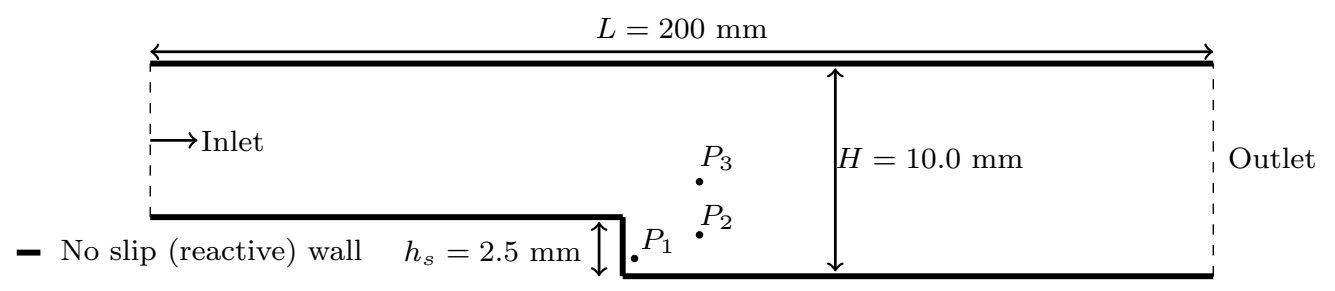

Fig. 4 2D Backward facing step computational domain. $P_{i}$ are the locations of the probes used in Figs. 5,7 and Fig. 11 $(P 1=(0.5,0.5), P 2=(4.0,1.5), P 3=(4.0,4.0) \mathrm{mm})$; the step and channel heights match the experimental values of Taylor et al. (2014). The thick black line denotes the solid boundary where boundary condition Eq. 9 is applied and activation of factor XII is allowed.

\begin{tabular}{ccc}
\hline Grid & $\Delta_{h}(\mu \mathrm{m})$ & Elements $\times 10^{3}$ \\
\hline Coarse & 70 & 367 \\
Medium & 60 & 484 \\
Fine & 52 & 646
\end{tabular}

Table 4 Mesh properties. $\Delta_{h}$ is the representative cell size computed as in Celik et al. (2008) and the total number of grid elements (triangles) are displayed.

\section{Results: Backward facing step}

\subsection{Flow Dynamics}

The velocity field reached a steady state solution before $1.0 \mathrm{~s}$ as shown in Fig. 5 for different spatial point probes located inside and outside the recirculation zone.

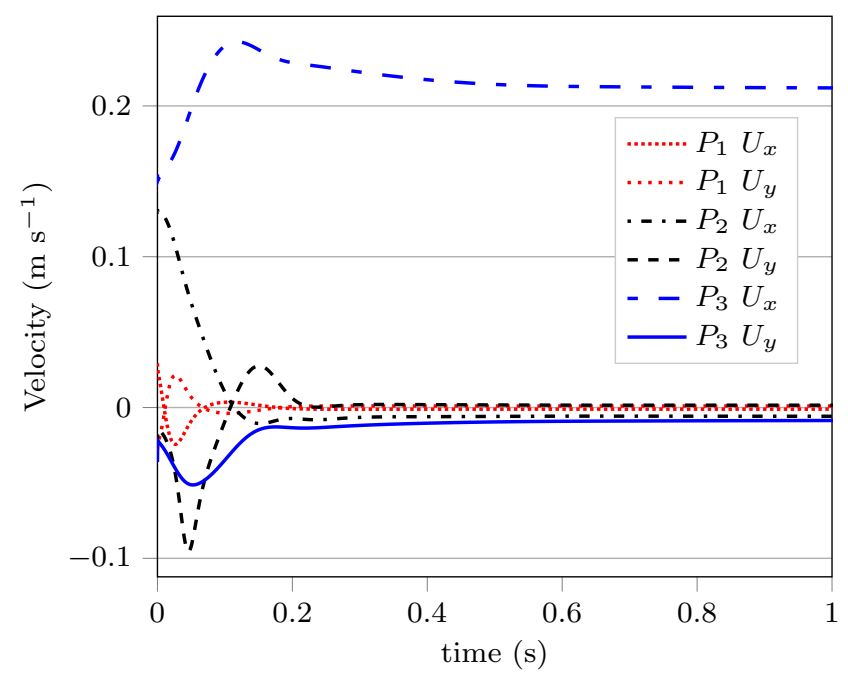

Fig. 5 Temporal evolution of $x$ and $y$ velocity components in different point probes.

Figure 6 shows the steady state velocity field as well as the streamlines that allow the visualization of recirculation region after the step. The reattachment length $X_{r}$ is in good agreement with the numerical and experimental results published in Kim and Moin (1985);

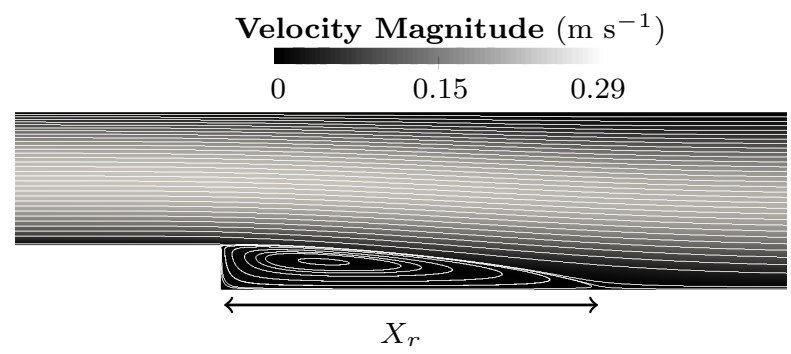

Fig. 6 Velocity magnitude with white stream lines. The reattachment length $X_{r}$ is shown.

Armaly et al. (1983) which lead to $X_{r}=8.9 h$. The comparison was performed for all the grids used in this study. The percentage error of the coarser mesh was $e_{r e l}=3.1 \%$ and for the medium and fine mesh of about $e_{\text {rel }}=2.9 \%$ which is a fair approximation considering the main objective of the current study.

\subsection{Coagulation cascade}

In order to quantify the outcome of the coagulation cascade, the value of total thrombin is considered $\left(I I_{a}+\right.$ $1.2 m I I_{a}$ is a quantitative measure of thrombin activity in the system, according to Hockin et al. (2002)). First, a mesh convergence study was performed using the mean value of the total thrombin in the computational domain. The methodology published in Celik et al. (2008) was use. The convergence grid index is used to quantify the distance of a computed value (simulation result) to the asymptotic value estimated by an extrapolation method using the different levels of discretization. For the finest mesh the convergence grid index obtained was $C G I_{\text {finest }}=0.41 \%$ with a relative extrapolated error of $e_{e x t}=0.33 \%$, the quantity used for the analysis was the total thrombin concentration value of a point probe inside the recirculation region. These values ensure that the total thrombin concentration field would not substantially change with even a finer mesh. The surface rate used for the mesh convergence study was $k_{s}=5.0 \times 10^{-4} \mathrm{~m} \mathrm{~s}^{-1}$, in order to 
reduce the computational cost of the study.

Figure 7 shows the time evolution of total thrombin at point probes $P_{1}, P_{2}$ and $P_{3}$ (see Fig. 4 for the location of the probes). The time response is characterized by a lag time of about $100 \mathrm{~s}$. Then, a propagation phase is observed until a small peak which evolves into a more stable plateau close to the inlet value of $I I=1.4 \mu \mathrm{M}$.

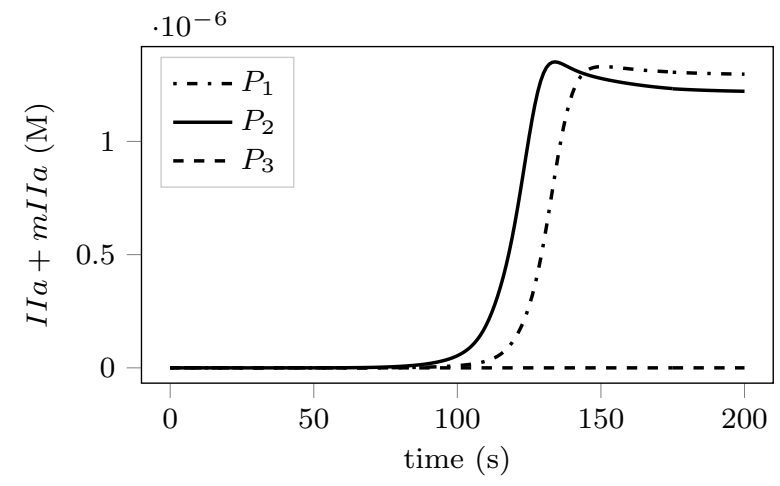

Fig. 7 Time evolution of $I I_{a}+1.2 m I I_{a}$ for each point probe (see Fig. 4). $P_{1}$ is near the wall region, $P_{2}$ inside the recirculation zone and $P_{3}$ outside the recirculation region.

Figure 8 shows the concentration field evolution of total thrombin at different time values. It can be observed that the region of largest total thrombin concentration is located inside the recirculation zone downstream of the step. Large concentrations of total thrombin extend further downstream at the lower wall.

The local concentration of coagulation factors inside the recirculation region is shown in Fig. 9. The plot is extracted along a profile located at the half height of the step $\left(h_{s}\right), y=1.25 \mathrm{~mm}$. It can be observed that the largest concentration of total thrombin is located at the step wall.

Thrombin concentration levels at the recirculation region are large enough to form a stable thrombus (Biasetti et al. (2012)). Furthermore, the zone with the largest concentration of thrombin aligns well with the experimental zone in which thrombus is formed (Taylor et al. (2014)).

In macroscopic applications, flow metrics such as the wall shear stress are commonly used to evaluate the thrombogenic performance of medical devices. Such flow quantities are practical to use due to their low computational cost compared to a reacting flow study. It is thus interesting to investigate how the outcome from the contact system and the wall shear stress correlate. Figure 10 shows a scatter plot of the wall shear stress
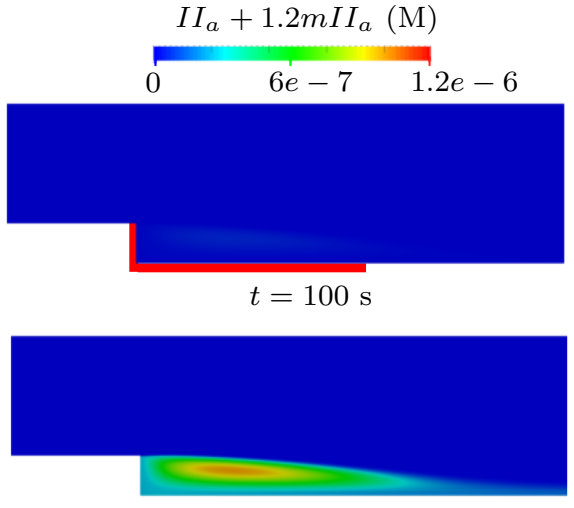

$t=125 \mathrm{~s}$

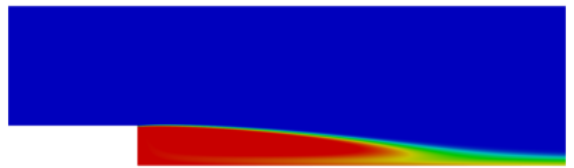

$t=150 \mathrm{~s}$

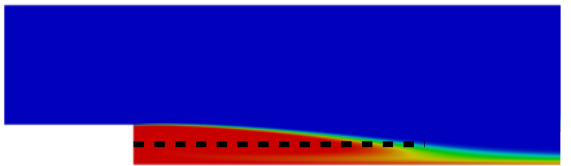

$t=175 \mathrm{~s}$

Fig. 8 Total thrombin concentration field at $t=100,125$ 150 and $175 \mathrm{~s}$. The black dashed line represents over which total thrombin is plotted in Fig. 9. The red line $(t=$ 100) represents approximately the experimental wall region of thrombus formation of Taylor et al. (2014).

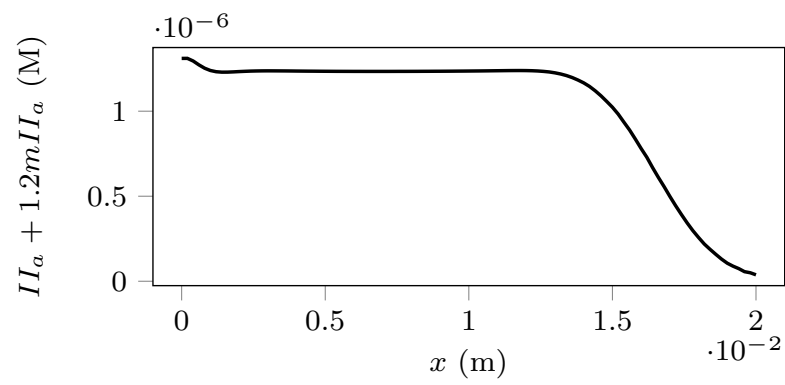

Fig. $9 I I_{a}+1.2 m I I_{a}$ concentration in the recirculation zone.

against the near wall concentrations of factors $X I I_{a}$ and $X I_{a}$. The quantities were normalized by the maximum wall shear stress and maximum concentrations of species $X I I_{a}$ and $X I_{a}$. Factor $X I I_{a}$ is clearly not correlated to WSS. Even though a clear correlation is not observed neither for XIa, this factor takes its largest values in areas where WSS is small. At the end, these results demonstrate that accounting for the contact system generates a physical behaviour which cannot be properly represented by WSS-based flow analysis. 

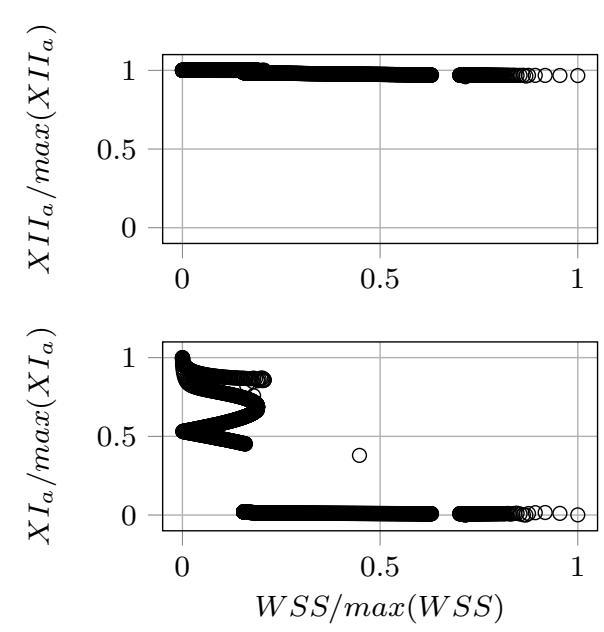

Fig. 10 Scatter plot of wall shear stress and wall concentrations of factors $X I I_{a}$ and $X I_{a}$.

\subsection{Parametric studies}

\subsubsection{Influence of surface kinetics $k_{s}$}

As already mentioned, there is a lack of reliable data about the surface reaction rate ( $k_{s}$ contact activation) and this quantity may depend on the material type. The nominal value $\left(k_{s}=5.0 \times 10^{-7} \mathrm{~m} \mathrm{~s}^{-1}\right)$ was obtained from the conversion of the volume reaction rate published in Chatterjee et al. (2010), as previously explained, using a dimensional analysis that involves the characteristic volume and the active surface from the experimental set up reported in Chatterjee et al. (2010). To investigate how the outcome of the simulations depends on this parameter, a parametric study was performed. Six values were considered in the range $k_{s}=$ $\left[5.0 \times 10^{-5}, 5.0 \times 10^{-10}\right] \mathrm{m} \mathrm{s}^{-1}$ spanning six orders of magnitude. Figure 11 shows the evolution at point probe $P_{1}$ of factors $X I I_{a}, X I_{a}$ and $I I_{a}+1.2 m I I_{a}$ over time. In addition, inserts in the plots display the variation of specific times of interest with respect to $k_{s}$. For $I I_{a}+1.2 m I I_{a}$ and $X I I_{a}$, the inflection point of the time evolution graphs is extracted by fitting a hyperbolic tangent function. For factor $X I_{a}$, the insert shows the time at which the maximum concentration is reached. The thrombin generation starts earlier as the parameter $k_{s}$ is increased. However, while five orders of magnitude are explored for the value of $k_{s}$, the impact on the thrombin generation time is moderate and the dependence appears to be logarithmic. In addition, the total thrombin concentration fields (steady state solution) were compared, showing no significant difference. It can be observed from the evolution of factors $X I I_{a}$ and $X I_{a}$ that the time delay present in the dynamics of these factors follow the same trend as the one observed for $I I_{a}+1.2 m I I_{a}$. In other words, the initial delay in the activation of factor $X I I$ is directly responsible for the delay in thrombin generation. While the influence of $k_{s}$ on the time needed for generation of factor $X I I_{a}$ is very large, this time has to be compared with the total time needed for the rest of the coagulation cascade, which is 10 times larger in the present case. It is important to note that when $k_{s}$ was set to zero the thrombin concentration levels remained close zero (not shown).
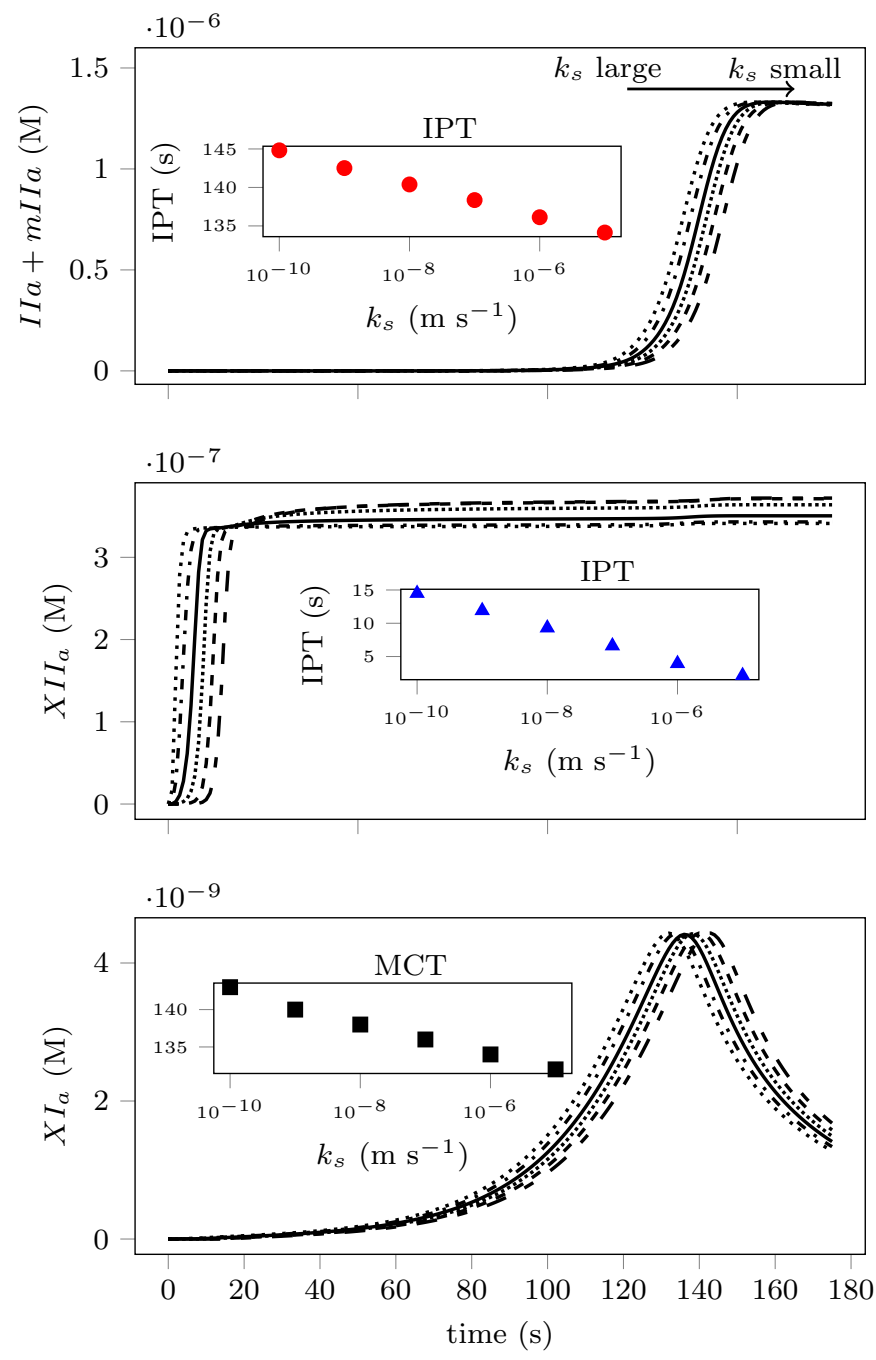

Fig. 11 Time evolution at point probe $P_{1}$ of total thrombin (upper), activated factor $X I I$ (medium) and activated factor $X I$ (bottom). $k_{s}=5 \times 10^{-5}(\cdots \cdots), k_{s}=5 \times 10^{-6}(\cdot-\cdot)$, $k_{s}=5 \times 10^{-7}(-), k_{s}=5 \times 10^{-8}(\cdots \cdots \cdots), k_{s}=5 \times 10^{-9}$ $(--), k_{s}=5 \times 10^{-10}(--)$. The insets of $I I_{a}+1.2 m I I_{a}$ and $X I I_{a}$ factor show the Inflection Point Time (IPT) for each $k_{s}$ value. For factor $X I_{a}$, the inset displays the maximum concentration time (MCT) as a function of $k_{s}$. 
3.3.2 Parametric study: Influence of factor XII inlet boundary condition.

A parametric study on the influence of of the concentration of factor $X I I$ at the inlet of the domain was also performed. This allows to evaluate the variability of the results to different samples with deficient factor $X I I$. Table 5 shows the four different concentration values that were used for this purpose.

\begin{tabular}{cc}
\hline Case & XII $(\mu \mathrm{M})$ \\
\hline 1 & 0.306 \\
2 & 0.272 \\
3 & 0.17 \\
4 & 0.102 \\
\hline
\end{tabular}

Table 5 Factor XII inlet concentrations used in the parametric study. These values correspond to $90 \%, 70 \%, 50 \%$ and $30 \%$ of the nominal concentration reported in Table 3 .

Figure 12 shows the time evolution of factors $X I I_{a}$, $X I_{a}$ and $I I_{a}+1.2 m I I_{a}$ at the point probe $P_{1}$. For each case, factor $X I I_{a}$ reaches a plateau equal to the inlet concentration of factor $X I I$, as expected. The results show longer lag times for thrombin propagation as the concentration of factor $X I I$ is decreased. Interestingly the maximum production of factor $X I_{a}$ is increased as factor XII concentration is reduced. This can be explained by the fact that a larger proportion of the available factor $X I$ is activated by factor $I I_{a}$ (reaction 19) in this case, instead of being generated by factor $X I I_{a}$ (reaction 20). This behavior is confirmed by the time evolution of the source term of reactions 19 and 20 (not shown) and is also coherent with the reverse rate of reaction 19 ( $k_{33}$ in Table 1$)$ being very small compared to the reverse rate of reaction 20 ( $k_{36}$ in Table 1$)$. The results suggest that a small amount of activated factor $X I I$ (contact activation) is sufficient to initiate the thrombin propagation phase.

\section{Discussion and Conclusion}

Previous studies have modeled device-related thrombosis with an approach focussing on platelet activity (Goodman et al. (2005); Taylor et al. (2016)). However, the contact activation system is a key component of the thrombotic network (Larsson et al. (2014); Jaffer et al. (2015)) which up to date has not been included in a complete model of thrombus formation. The current model aims to serve as a first step towards the integration of the contact activation in a complete macroscopic
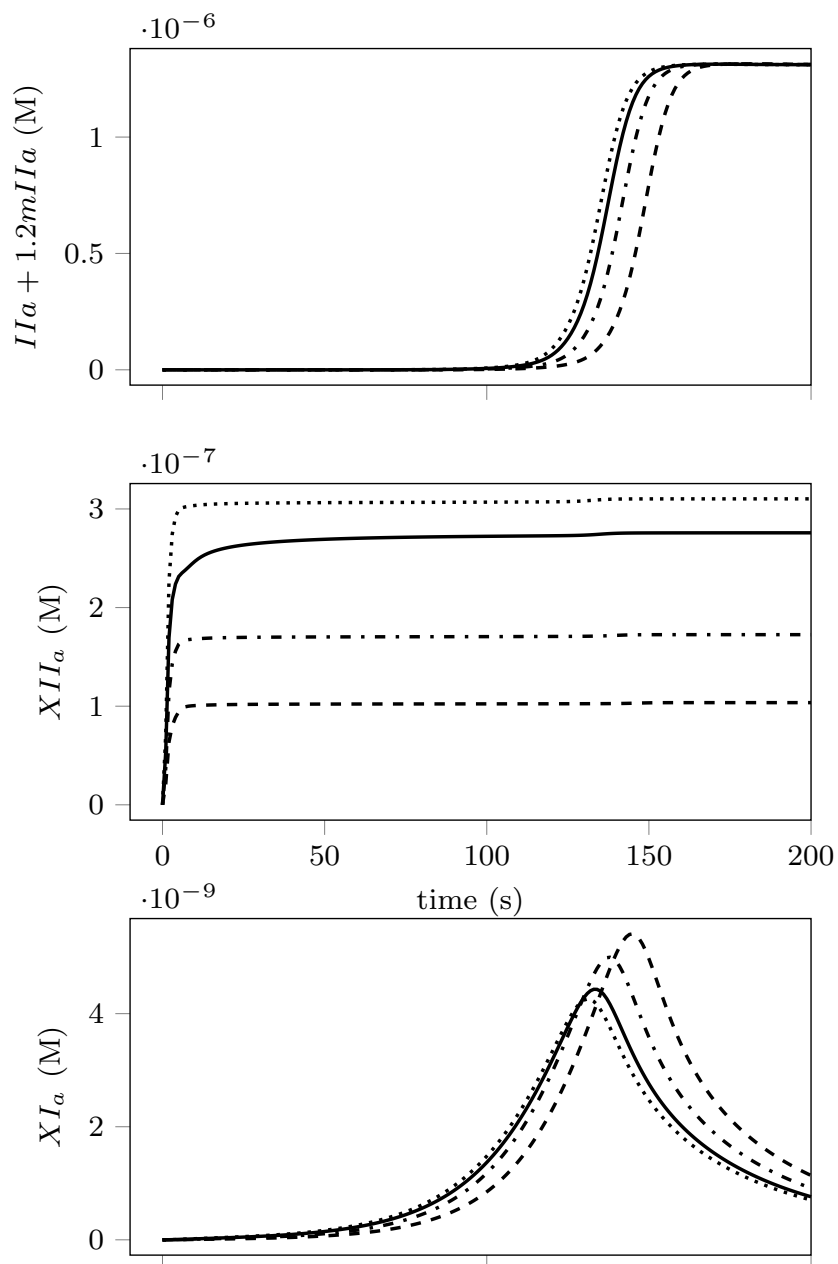

Fig. 12 Time evolution of point probe $P_{1}$ for total thrombin (upper), activated factor $X I I$ (medium) and activated factor $X I$ (bottom) at point probe $P_{1}$. The inlet and initial concentrations are $X I I_{90 \%}=0.306 \mu M(---), X I I_{70 \%}=0.272$ $\mu M(\cdot-\cdot), X I I_{50 \%}=0.17 \mu M(-), X I I_{30 \%}=0.102 \mu M$ $(\cdots \cdots)$.

thrombus formation model specific to medical devices. The current model was applied to a backward facing step configuration typical of medical devices.

One of the main features of the model is that it allows the initiation of the coagulation reactions without an a priori knowledge of the thrombotic zone. The results show that a significant amount of thrombin is generated in the recirculating region behind the backward facing step. This could be explained by comparing the characteristic residence and chemical times. Large residence time at the recirculating regions enhances the thrombin production reactions. Qualitatively, the model leads to higher total thrombin concentrations in regions where the thrombus formation was observed experimentally. The results also show a poor correlation between wall shear stress and near wall concentrations of factor $X I_{a}$, 
and no correlation for factor XIIa that is present all along the device surface. This suggests that the WSS should be complemented with other flow metrics that quantify diffusive and convective transports to characterize the thrombogenic performance of medical devices.

Molecular diffusivity of the species has been modified for numerical reasons (from $D_{i} \sim 10^{-11} \mathrm{~m}^{2} \mathrm{~s}^{-1}$ to $D_{i} \sim 10^{-8} \mathrm{~m}^{2} \mathrm{~s}^{-1}$ ), because the proper resolution of the species boundary layer would make the calculations prohibitive. However, it is most probably that if this modification has a consequence, it is only in the sense of underestimating the thrombin generation by the contact system. First, in the main stream, convection clearly dominates, the Peclet number is of the order $10^{7}$ and $10^{4}$ for the molecular diffusivity of $D_{i} \sim 10^{-11}$ $\mathrm{m}^{2} \mathrm{~s}^{-1}$ to $D_{i} \sim 10^{-8} \mathrm{~m}^{2} \mathrm{~s}^{-1}$ respectively. In contrast, in the recirculation zone the results are controlled by the competition between reactions and diffusion. Large $D_{i}$ values actually underestimate the local concentration of chemical species since they are diffused outside the recirculation region faster. The higher the diffusion coefficients, the lower the concentration of thrombin in the recirculation region. This is illustrated by two additional simulations with diffusion coefficient values of the order $D_{i} \sim 10^{-7} \mathrm{~m}^{2} \mathrm{~s}^{-1}$ and $D_{i} \sim 10^{-6} \mathrm{~m}^{2} \mathrm{~s}^{-1}$, which were performed to compare the concentration values inside the recirculation region. Figure 13 shows the underestimation of thrombin as the molecular diffusivity is augmented.

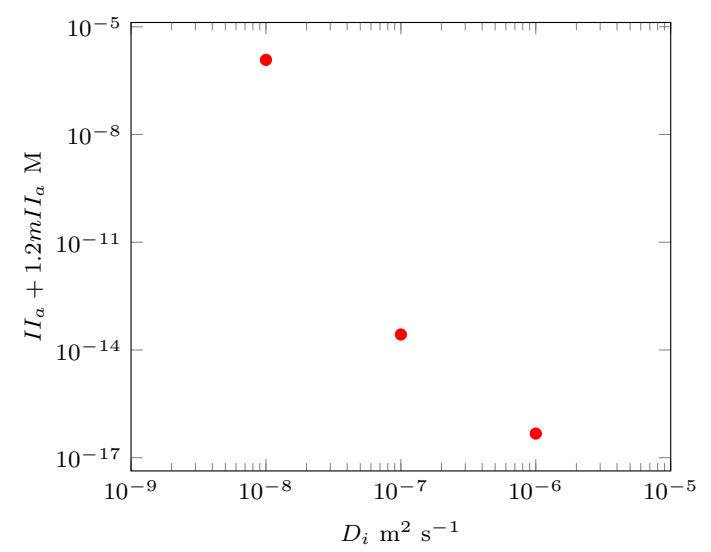

Fig. 13 Maximal total thrombin concentration inside the recirculation region for three orders of magnitude of the diffusion coefficients $\left(D_{i} \sim 10^{-8}, 10^{-7}, 10^{-6} \mathrm{~m}^{2} \mathrm{~s}^{-1}\right)$.

An interesting feature of the present model is that it includes a material property $k_{s}$ (typical to each medical device) which is a novel feature for thrombus predictive models representing the kinetics of the contact sur- face reaction. A parametric study was performed on $k_{s}$ showing a moderate influence of this parameter on the total thrombin generation, although it strongly modifies the generation time of factor $X I I_{a}$. This is because the characteristic time of this later mechanism (reaction 14 in Table 1) is very small compared to the whole time required to generate thrombin.

In the end, the current study focuses on the contact system as one potential player in thrombin production. This approach could complement more complex models that consider platelet activity, fibrin formation and fluid structure interaction of the growing thrombus, providing a proper initiation of the coagulation reactions in medical devices.

Acknowledgements We are grateful to the CONACyT, Mexico scholarship and the LabEx Numev (convention ANR-10LABX-20) for their financial support. Drs. Vincent Moureau and Ghislain Lartigue from CORIA laboratory, and the SUCCESS scientific group are acknowledged for providing the YALES2 solver which constitutes the basis of the YALES2BIO tool. Professor Jean-François Schved and MD. Muriel Giansily from the University Hospital of Montpellier are thanked for helpful discussions about the biological aspects of thrombosis. This work was performed using HPC resources from GENCI-CINES (Grants 2016-c2015037194 and 2017-A0020307194) and with the support of the High Performance Computing Platform HPC@LR.

Conflict of Interest: The authors declare that they have no conflict of interest.

\section{References}

Alemu Y, Girdhar G, Xenos M, Sheriff J, Jesty J, Einav S, Bluestein D (2010) Design optimization of a mechanical heart valve for reducing valve thrombogenicity - a case study with ats valve. Am. Soc. Artif. Intern. Org. 56(5):389-396

Anand M, Rajagopal K, Rajagopal KR (2003) A model incorporating some of the mechanical and biochemical factors underlying clot formation and dissolution in flowing blood. J. Theor. Med.5:183-218

Anand M, Rajagopal K, Rajagopal KR (2008) A model for the formation, growth, and lysis of clots in quiescent plasma. a comparison between the effects of antithrombin iii deficiency and protein c deficiency. J. Theor. Biol.253:725-738

Armaly BF, Durst F, Pereira JCF (1983) Experimental and theoretical investigation of backward-facing step flow. J. Fluid Mech.127:473-496

Basmadjian D, Sefton MV, Baldwin SA (1997) Coagulation on biomaterials in flowing blood: Some theoretical considerations. Biomaterials18:1511-1522

Biasetti J, Spazzini PG, Swedenborg J, Gasser TC (2012) An integrated fluid-chemical model toward modeling the formation of intra-luminal thrombus in abdominal aortic aneurysms. Front. Physiol.3(266)

Celik IB, Ghia U, Roache PJ, Freitas CJ, Coleman H, Raad PE (2008) Procedure for estimation and reporting of un- 
certainty due to discretization in cfd applications. J. Fluids Eng.130, article id 078001

Chan MY, Weitz JI, Merhi Y, Harrington RA, Becker RC (2009) Catheter thrombosis and percutaneous coronary intervention:fundamental perspectives on blood, artificial surfaces and antithrombotic drugs. J. Thromb. Thrombol.28:366-380

Chatterjee MS, Denney WS, Jing H, Diamond SL (2010) Systems biology of coagulation initiation: Kinetics of thrombin generation in resting and activated human blood. PLoS Comp. Biol.6(9)

Chnafa C, Mendez S, Moreno R, Nicoud F (2014a) The Cardio-Circulatory System : from Modeling to Clinical Applications, Springer, chap Using image-based CFD to investigate the intracardiac turbulence

Chnafa C, Mendez S, Nicoud F (2014b) Image-based largeeddy simulation in a realistic left heart. Comput. Fluids94:173-187

Chnafa C, Mendez S, Nicoud F (2016) Image-based simulations show important flow fluctuations in a normal left ventricle: What could be the implications? Ann. of Biomed. Eng.44(11):3346-3358

Fogelson AL (1992) Continuum models of platelet aggregation: Formulation and mechanical properties. SIAM J. Appl. Math.52(4):1089-1110

Fogelson AL, Guy RD (2008) Immersed-boundary-type models of intravascular platelet aggregation. Comput. Meth. Appl. Mech. Eng.197:2087-2104

Fogelson AL, Neeves KB (2015) Fluid mechanics of blood clot formation. Ann. Rev. Fluid Mech.47(1):377-403

Furie B, Furie BC (2008) Mechanisms of thrombus formation. New Eng. J. Med.359:938-949

Goodman PD, Barlow ET, Crapo PM, Mohammad SF, Solen KA (2005) Computational model of device-induced thrombosis and thromboembolism. Ann. of Biomed. Eng. 33:780-797

Gorbet MB, Sefton MV (2004) Biomaterial-associated thrombosis: roles of coagulation factors, complement, platelets and leukocytes. Biomaterials25:5681-5703

Gregory K, Basmadjian D (1994) An analysis of the contact phase of blood coagulation: Effects of shear rate and surface are intertwined. Ann. of Biomed. Eng.22:184-193

Hastings SM, Ku DN, Wagoner S, Maher OK, Deshpande S (2017) Sources of circuit thrombosis in pediatric extracorporeal membrane oxygenation. Am. Soc. Artif. Intern. Org. 63(1):86-92

Hellums JD (1994) 1993 whitaker lecture: Biorheology in thrombosis research. Ann. of Biomed. Eng.22:445-455

Hockin MF, Jones KC, Everse SJ, Mann KG (2002) A model for the stoichiometric regulation of blood coagulation. J. Biol. Chem.277(21):18,322-18,333

Jaffer IH, Fredenburgh JC, Hirsh J, Weitz JI (2015) Medical device-induced thrombosis: what causes it and how can we prevent it? J. Thromb. Haemost.13(Suppl. 1):72-81

Jetsy J, Yin W, Perrotta P, Bluestein D (2003) Platelet activation in a circulating flow loop: combined effects of shear stress and exposure time. Platelets14(3):143-149

Kim J, Moin P (1985) Application of a fractional-step method to incompressible Navier-Stokes equations. J. Comput. Phys.59:308-323

Kroll MH, Hellums JD, McIntire LV, Schafer AI, Moake JL (1996) Platelets and shear stress. Blood88(5):1525-1541

Kuharsky AL, Fogelson AL (2001) Surface-mediated control of blood coagulation: the role of binding site densities and platelet deposition. Biophys. J.80:1050-1074
Lanotte L, Mauer J, Mendez S, Fedosov DA, Fromental JM, Clavería V, Nicoud F, Gompper G, Abkarian M (2016) Red cells' dynamic morphologies govern blood shear thinning under microcirculatory flow conditions. Proc. Natl Acad. Sc. USA113(47):13,289-13,294

Larsson M, Rayzman V, Nolte MW, Nickel KF, Bjrkqvist J, Jms A, Hardy MP, Fries M, Schmidbauer S, Hedenqvist P, Broom M, Pragst I, Dickneite G, Wilson MJ, Nash AD, Panousis C, Renné T (2014) A factor xiia inhibitory antibody provides thromboprotection in extracorporeal circulation without increasing bleeding risk. Sci. Transl. Med.6(222)

Leiderman K, Fogelson AL (2011) Grow with the flow: A spatial-temporal model of platelet deposition and blood coagulation under flow. Math. Med. Biol.28:47-84

Mehra MR, Stewart GC, Uber PA (2014) The vexing problem of thrombosis in long-term mechanical circulatory support. J. Heart Long Transplant.33:1-11

Mendez S, Gibaud E, Nicoud F (2014) An unstructured solver for simulations of deformable particles in flows at arbitrary Reynolds numbers. J. Comput. Phys.256(1):465483

Michaelis L, Menten M, Johson K, Goodoy R (2011) The original michaelis constant: translation of the 1913 michaelismenten paper. Biochemistry-US50(39):8264-8269

Moureau V, Domingo P, Vervisch L (2011) Design of a massively parallel CFD code for complex geometries. Comp. Rend. Méc.339(2-3):141-148

Neeves KB, Illing DAR, Diamond SL (2010) Thrombin flux and wall shear rate regulate fibrin fiber deposition state during polymerization under flow. Biophys. J.98:13441352

Ngoepe MN, Ventikos Y (2016) Computational modelling of clot development in patient-specific cerebral aneurysm cases. J. Thromb. Haemost.14(2):262-272

Nobili M, Sheriff J, Morbiducci U, Redaelli A, Bluestein D (2008) Platelet activation due to hemodynamic shear stresses: Damage accumulation model and comparison to in vitro measurement. Am. Soc. Artif. Intern. Org. 54(1):64-72

Piatti F, Sturla F, Marom G, Sheriff J, Claiborne TE, Slepian MJ, Redaelli A, Bluestein D (2015) Hemodynamic and thrombogenic analysis of a trileaflet polymeric valve using a fluid-structure interaction approach. J. Biomech.48(13):3650-3658

Rice NT, Szlam F, Varner JF, Bernstein PS, Szlam AD, Tanaka KA (2016) Differential contributions of intrinsic and extrinsic pathways to thrombin generation in adult, maternal and cord plasma samples. PLoS One11(5):e0154,127

Seo JH, Abd T, George RT, Mittal R (2016) A coupled chemo-fluidic computational model for thrombogenesis in infarcted left ventricles. Am. J. Phys.310(11):H1567H1582

Shadden SC, Hendabadi S (2013) Potential fluid mechanic pathways of platelet activation. Biomech. Model. Mechanobiol.12:467-474

Sigüenza J, Mendez S, Nicoud F (2017) How should the optical tweezers experiment be used to characterize the red blood cell membrane mechanics? Biomech. Model. Mechanobiol.16:1645-1657

Sorensen EN, Burgreen GW, Wagner WR, Antaki JF (1999) Computational simulation of platelet deposition and activation: I. model development and properties. Ann. of Biomed. Eng.27:436-448 
Taylor J, Witmer K, Neuberger T, Craven B, Meyer R, Deutsch S, Manning KB (2014) In vitro quantification of time dependant thrombus size using magnetic resonance image and computational simulations of thrombus surface shear stresses. J. Biomech. Eng.136(7)

Taylor JO, Meyer RS, Deutsch S, Manning KB (2016) Development of a computational model for macroscopic predictions of device-induced thrombosis. Biomech. Model. Mechanobiol.15(6):1713-1731

Yazdani A, Li H, Humphrey JD, Karniadakis GE (2017) A general shear-dependent model for thrombus formation. PLoS Comp. Biol.13(1):e1005,291

Young ME, Carroad PA, Bell RL (1980) Estimation of diffusion coefficients of proteins. Biotechnol. Bioeng.22(5):947-955

Zhu D (2007) Mathematical modeling of blood coagulation cascade: kinetics of intrinsic and extrinsic pathways in normal and deficient conditions. Blood. Coagul. Fibribolysis.18:637-646

Zmijanovic V, Mendez S, Moureau V, Nicoud F (2017) About the numerical robustness of biomedical benchmark cases: Interlaboratory FDA's idealized medical device. Int. J. Numer. Meth. Biomed. Eng.33(1):e02,789:1-17 